\title{
Creativity in Humans, Robots, Humbots
}

\section{Todd Lubart1, 2, Dario Esposito', Alla Gubenko3, and Claude Houssemand 3}

1 LaPEA, Université de Paris and Univ. Gustav Eiffel, F-92100 Boulogne-Billancourt, France 2 HSE University, Moscow, Russia

3 Department of Education and Social Work, Institute for Lifelong Learning and Guidance, University of Luxembourg, Luxembourg

\begin{abstract}
This paper examines three ways that robots can interface with creativity. In particular, social robots which are designed to interact with humans are examined. In the first mode, human creativity can be supported by social robots. In a second mode, social robots can be creative agents and humans serve to support robot's productions. In the third and final mode, there is complementary action in creative work, which may be collaborative co-creation or a division of labor in creative projects. IIlustrative examples are provided and key issues for further discussion are raised.
\end{abstract}

\section{KEYWORDS:}

creation process, human-robot interaction, human-robot co-creativity, embodied creativity, social robots

Note: The article was prepared in the framework of a research grant funded by the Ministry of Science and Higher Education of the Russian Federation (grant ID: 075-15-2020-928).

\section{Article history:}

Received: June 20, 2021

Received in revised from: July 9, 2021

Accepted: July 9, 2021

ISSN 2354-0036

DOI: 10.2478/ctra-2021-0003

\section{Corresponding author at:}

Todd Lubart

E-MAIL: todd.lubart@u-paris.fr 


\section{INTRODUCTION}

Robots are agents equipped with sensors, actuators and effectors which enable them to move and perform manipulative tasks (Russell \& Norvig, 2010). The term 'Robot' stems from the Slavic word «robota», referring to obligatory work such as the work required of peasants in a feudal society. In 1920, Čapek, a Czech author, wrote a fictional story about « robots », in which soul-less human-like machines worked in factories doing monotonous, repetitive tasks. In most classic visions of robots, they are electromechanical systems programmed to do specific repetitive jobs.

In contrast with the initial vision of robots accomplishing boring, routine tasks, the rise of advanced technology, more specifically machine learning, now allows us to envision that machines may become creative agents (Gubenko et al., 2021 ; OECD, 2021). Creativity is defined as the capacity to generate novel, original output (work, ideas, etc.) that is meaningful and valuable in a defined context (Runco \& Jaeger,2012). Although this topic could be considered timely and new, there is a long history. Indeed, since thousands of years, people have considered the idea that mechanical automata could be created to resemble humans and animals, and do complex tasks such as playing a musical instrument. Kantosalo et al. (2021) illustrate, through their analysis of 18th century literary works, the idea that machines can have creative ability, and can support human creativity or cocreate with humans.

In the mid 20th century, as depicted in early science fiction films, robots, such as Robbie the Robot in Forbidden Planet, were envisioned as agents who provided support in terms of advanced cognitive capacities. In Star Wars, R2D2 and C3PO illustrate respectively non-humanoid and humanoid intelligent agents. Both of them remain somewhat primitive compared to Mr Data on the most recent version of Star Trek. Data is an extremely sophisticated robot that looks very much like a human, has advanced capacities that go beyond those of humans in some ways, thinks in some non-human ways, and integrates well into human society. Mr. Data is not human, he is not made of human material and seems not to have emotions. He does, however, have some original ideas that prove to be very useful to solve complex problems encountered in Star Trek space missions. Data might be considered creative, at least by human standards. This short «family portrait» illustrates several «social» robots. They are social because one of their key design features is to interact with humans.

Social robots can be contrasted with non-social robots, designed to accomplish tasks without any interaction with humans. Industrial robots such as the car welding robots could be an example of non-social robots (U.N. and I.F.R.R., 2002). These robots are designed to automatize routine mechanical tasks and thus, are more likely to replace jobs that do not require creativity (OECD, 2019). Other robots in this category are those designed for hazardous environments, such as robots that clean-up nuclear waste or navigate abandoned mines (Thrun et al., 2003; Thrun, 2004). When a human interacts with this kind of robot, it means generally that the robot needs maintenance. More recently, a number of non-social robots are commercially available as personal service robots. A domestic robot lawn mower that can optimize the specialized task of cutting grass in a person's yard, a robot to vacuum one's home or a robot that serves food in a restaurant are examples. 


\section{SOCIAL ROBOTS AND CREATIVITY}

According to Dautenhahn and Billard (1999, as cited in Fong et al., 2002 p. 2-3) social robots are "embodied agents that are part of a heterogeneous group: a society of robots or humans. They are able to recognize each other and engage in social interactions, they possess histories (perceive and interpret the world in terms of their own experience), and they explicitly communicate with and learn from each other". In the early days of social robotics, engineers tended to copy the humanoid character for robots on the assumption that humans are more willing to communicate with what looks like them or with beings close to their daily life than with objects (Duffy, 2003). This anthropomorphism of social robots was quickly abandoned as research showed that the strict resemblance between humans and robots could make their interaction problematic (MacDorman, 2019; De Graaf et al., 2015), referring for example to the concept of Uncanny Valley, introduced by Masahuro Mori in the 1970s, which describes the negative attitude of humans to very lifelike humanoids (Mori et al., 2012). Thus, the social character of robots lies not only in their functionality but also in their integration into the social sphere of humans as recognized non-human social entities (Leite et al., 2013; Höflich \& El Bayed, 2015; Magnenat-Thalmann et al. 2016; Vincent et al., 2015).

A more recent definition by Jones (2017) emphasizes social robot's responsiveness and ability to engage in dialogic action with humans. Three aspects are important with regard to creativity. First, robots are physically embodied agents. The embodiment of artificial agents enables physical interaction, which is a valuable asset compared to computers, tablets, or other virtual agents. Specifically, in contrast to "disembodied" models of creativity that were focused on the 'problemsolving mind' (Dautenhahn, 2007), physical embodiment of robots allows to reframe the problem and investigate how creativity emerges from robot's actions and bodily engagements with the environment. In terms of human-robot interaction, robots physical presence enables real-life collaborations with humans, as embodied artificial agents tend to be associated with greater human engagement because artificial agents are viewed as social actors rather than "tools" (Seerbeck et al., 2010; Zawleska \& Duffy, 2015; Belpaeme et al., 2018).

The second point, robots' social situatedness, implies that robots are surrounded by a social environment and have to maintain social interactions (Fong et al., 2002; Dautenhahn et al. 2002; Lindblom \& Ziemke, 2003). In contrast to some early computational models of creativity which aimed to autonomously generate a creative product (Hoffman, 2016), the field of human-robot interaction overcomes a relatively narrow focus on individuals and puts creativity in a social context. This aspect is particularly relevant in the sociocultural perspective where creativity is viewed as an inherently social construction that is distributed between the creator, the audience and sociocultural context (Csikszentmihalyi, 1999; Engeström, 2001; Glăveanu et al., 2020).

Drawing upon Gibson's (1986) work on affordances and Hutchin's (1995) ideas of distributed cognition, Glăvenau (2012; 2013) has defined creativity as a process of perceiving, exploiting affordances, and generating novel artifacts during socially and materially situated activities. As such, research on social robots constitutes an excellent case for creativity researchers not only to 
study human-robot co-creation, but also the processes of robot's internalization and externalization (Leont'ev, 1978; Engeström \& Miettinen, 1999; Bruner, 1996) of human culture. The question of how robots explore and exploit affordances and could be 'enculturated' through social interactions with humans is being investigated in developmental and cognitive robotics (Zlatev, 2001; Smith \& Gasser, 2005; Asada et al. 2009; Lungarella et al., 2004; Otero et al., 2008 ; Min et al., 2016; Cangelosi \& Schlesinger, 2018; Guerin \& Ferreira, 2019).

Finally, a third point is that social robots possess some analogues of human perceptual abilities: vision, hearing, proprioception, etc. Arguably, robots endowed with human-like sensorimotor skills hold potential to instantiate Ventura's (2016) highest level of an artificial system's creativity, in which robots create artifacts based on what they see, hear, and experience. Robots' perceptual endowments provide as well an opportunity to investigate how creativity is grounded in perception and clarify questions that are of particular relevance to creativity: how do we extract meaning from motorsensory experiences and arrive to abstract mental representations? How flexible representations are born? What is the mechanism underlying sudden representational changes?

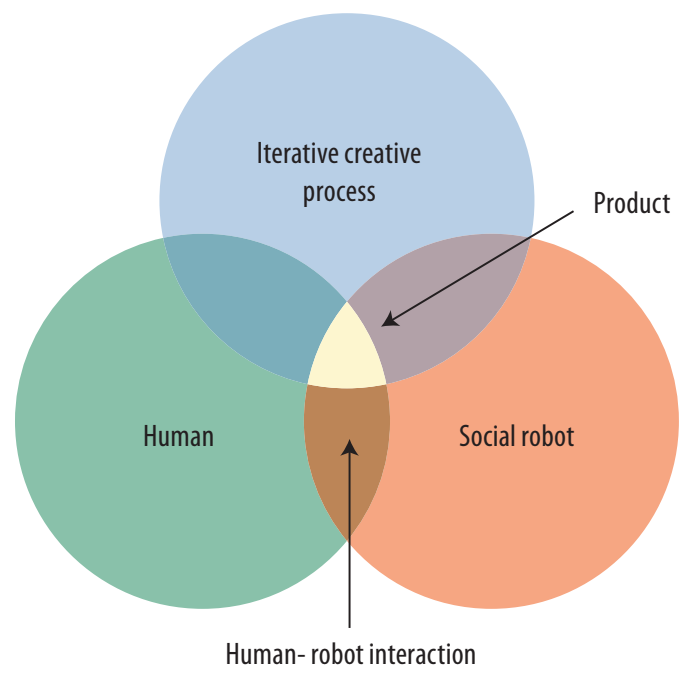

Figure 1. Human-robot co-creativity

As we have argued elsewhere (Gubenko et al., 2021) one particularly insightful line of research is using embodied agents as a testbed to emulate the creative process and verify existing models of creativity (see Dautenhahn, 2007). In this paper, we will complement this discussion by considering the potential of human-robot interaction (HRI) in the production of a creative outcome (Figure 1). In what follows we will consider three different positions in human-robot cocreation: 1) robots as a support for human creativity; 2) humans as a support for robotic creativity; 3) human-robot (humbot) co-creation. Specifically, we will address the following questions: can social robots support humans in the development of creative products? Inversely, what could be the role of humans in the robotic creative process? Finally, can social robots engage in a dialogic and cyclic creative process with humans and contribute to the emergence of a valuable and useful product? 


\section{HUMAN CREATIVITY SUPPORTED BY ROBOTS}

In this category, the focus is human creativity or human-centred HRI (human-robot interaction) as it has been defined by Dautenhahn (2007). In several projects, robots were designed to interact with humans engaged in the creative process. The common denominator is the use of a robot agent to provide some input to human creative endeavors. For example, in a storytelling task, a human may start to generate a story. Then, a social robot reacts and provides a cue. This stimulates the person to continue the story, in response to the social robot's behavior. To illustrate this line of work, consider the robot named YOLO (your own living object), a non-anthropomorphic robot to stimulate creativity in children, designed to counteract the developmental slump of creativity that may occur around the age of 8 (Alves-Oliveira et al., 2016, 2018, 2019, 2020). YOLO's intervention enables new directions to be activated in stories told by children. Two techniques are used. First, there is the "contrast" technique which provides children with stimuli that are not related to the story that is being told. Second, there is the "mirror" technique which allows to stimulate convergent thinking by providing stimuli that are connected to the story the children are exploring (Smith, 1998; Alves-Oliveira et al., 2018).

The example of YOLO could be extended. Imagine that the social robot may ask a series of questions to the child, such as, «will other children think this is original?» or «can you add something to make the story more unusual?». The social robot may be able to catch some semantic elements of the story, compare it to a database (like in Toubia et al., 2021), and provide feedback on the story's originality and potential creative success. In the musical domain, a human could play on a xylophone and then a robotic xylophone would play back the music with some added variations, inviting a human creator to reflect on his or her composition (Addesi \& Pachet, 2005, Pachet, 2003). Alternatively, the social robot could provide some «random» input, such as a word, a sound, a picture that the human may find interesting and try to integrate in the work in progress. In this perspective, the social robot is essentially an assistant to foster the human creative process (see Figure 2).

In this vein, Kahn et al. $(2012,2016)$ conducted a study showing how interacting with a social robot can help stimulate creativity, compared to the absence of interaction. In their study, the researchers were searching for a difference in creativity under two experimental conditions. In the first condition participants had to produce the most creative ideas in a small Zen rock garden setting with the help of the robot Robovie. The robot's role was to encourage the participants by verbally interacting and proposing different kinds of sources from the internet such as images or videos to stimulate and support participants' ongoing creative ideation. In the second condition, the participants were asked make the same creative efforts without having a direct interaction with the robot which was replaced by the use of a powerpoint. Results showed that verbal and physical interactions with social robot could enhance human creativity in terms of the creative process by doubling the engagement time in the creative task, as well as in terms of the creative product - by increasing the number of different creative ideas. 


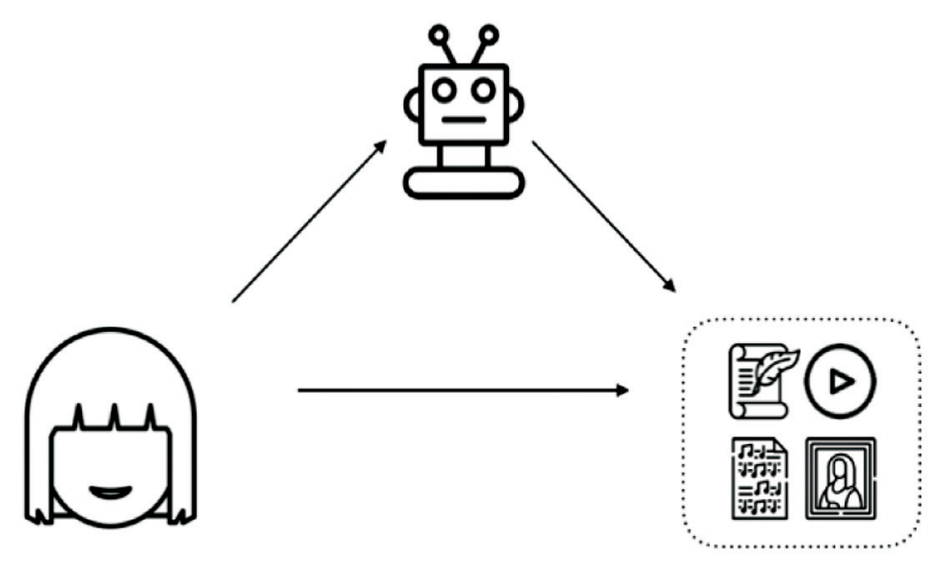

Figure 2. Robot as a support for human creativity

This very promising line of work can be even further developed if we consider how social robots could implement creative thinking techniques. Although a simple computer interface could to some extent provide the same stimulation, the animated, physically present social robots may significantly enhance the engagement with the artificial agent and facilitate the effect. A robot might suggest, for example, to formulate problems, activate relevant information (or help to find this information), to look for metaphors or pay attention to environmental affordances, or suggest to evaluate the intermediate result from different perspectives. Also, it could motivate humans to engage in alternate cycles of generation and exploration. In addition to direct help provided during the human creative process, social robots have been used as tools to promote the learning of certain types of creativity-relevant behavior such as curiosity, perseverance or attention (Belpaeme et al., 2018; Gordon et al., 2015; Park et al., 2019). One eloquent example is the study by Park and collaborators (2017), where a peer-like social robot helped foster a growth mindset in young children during a puzzle-solving game. According to authors, growth mindset is considered to include intrinsic curiosity, perseverance and grit. Two conditions, a robot with a growth mindset versus a robot with a neutral mindset, were created to investigate the differences in self-reported behaviors and engagement during the task. The children with the growth mindset robot showed consciousness of their own growth mindset and a greater engagement during the challenging task as compared to the condition where they were playing the game with a neutral mindset robot. These results support the idea of extending the expressive cognitive architecture of the robot to include more cognitive aspects of the learning process (see Chen et al., 2020).

In a rather different approach, Ali et al. (2019) examined the impact of interacting with creative or non-creative robots in a game-play context using the robot named Jibo (https://www.jibo.com/). In this work, we can observe the possibility for social robots to show creative abilities which in turn can stimulate the creative abilities of children ranging from 6 to 10 years old. In the game, robots provide stimulation, through their own creative (or non-creative) input to affect children's creative ideation. According to the authors, the competitive spirit of the game could be stimulating, but a cooperative game involving a social robot and humans could also be envisioned. Thus, social 
robots may potentially support the development of creativity when they serve as sparring partners in educational game settings. Although this line of research is promising, more research is needed to better control and define creativity-related variables.

\section{ROBOT CREATIVITY SUPPORTED BY HUMANS}

In this category, our analysis is concerned by robot-centred HRI (Dautenhahn, 2007) and emphasizes robots that produce work that is, by design, supposed to be original and potentially valuable (Oliveira, 2020; Pan et al., 2010; Peng et al., 2015). The most classic cases in this category are robot artists and composers. For example, case studies present different robots with humanoid appearances (Kato et al., 1987; Calinon et al., 2005) or not (Gazeau \& Zeghloul, 2012; Tresset \& Leymarie, 2013; Luo \& Liu, 2018) which succeed in producing drawings of portraits of human beings. An example of a creative robot with humanoid appearances is the one used in the study of Calinon and collaborators (2012), in which a humanoid robot holds a pen in its hand and can draw an image of any person who stands in front of it thanks to face detection and image processing systems. With regards to nonhumanoid robots, Luo and Liu (2018) made a case study of a robot capable of painting like a human cartoonist. In this study, a robotic arm is able to transform the face detected in a human picture into a cartoon facial type by adding hand-made strokes and a variety of colors coming from the original picture. The robot artist seeks to represent the subject (a person), taking into account the unique details of the person who poses thanks to neural network techniques applied to face verification (Zagoruyko \& Komodakis, 2015). The robot has a certain style and each drawing is new, unique and meaningful because it resembles, to some extent, the person who posed. The value is appreciated by the human who posed.

In another example, a robot musician can generate a jazz composition by respecting a set of initial coded rules, and generating some random notes at certain moments in the sequence (Hoffman \& Weinberg, 2011). Then the rule constraints lead the robot composer to complete the new musical sequence in a harmonious way. The robot may look like a «musician» and actually plays a musical instrument. Each composition is original, and the audience can find the music aesthetically pleasing and perhaps interesting and valuable in terms of an expanded jazz repertoire.

One issue that can be raised concerning this category is the extent to which it is appropriate to consider that these robots are actually social. From one perspective, although the robots generate productions, there is not really any specific social interaction with humans. In the example of the drawing robot, the robot has no real social interaction with the human. The robot could make a portrait of a plastic manikin, or a pineapple with eyeglasses, as far as it is concerned.

From an alternative perspective, however, the use of the term social robot is appropriate. These robots are designed to produce work that is destined for human appreciation, and the productions have value when the human interacts with them. Beyond the simple novelty of the productions for the given robot artist (compared to the robot's earlier works), the novelty of the production 
is estimated by human judges who evaluate it with respect to other works, essentially from the human-produced repertoire. Thus, the robot is, in this way, a social agent. Potentially, in advanced systems, the robot could learn from human feedback about the creative success of its' productions and improve itself to generate more creative content in the future (see Figure 3).

Another fascinating and nascent line of research that falls in the same category is research on robots' dealing with novelty from humans demonstrating a task. Fitzgerald et al. (2017) have addressed the issue by studying how robots could learn to transfer a task into a new environment by relying on human teacher's assistance.

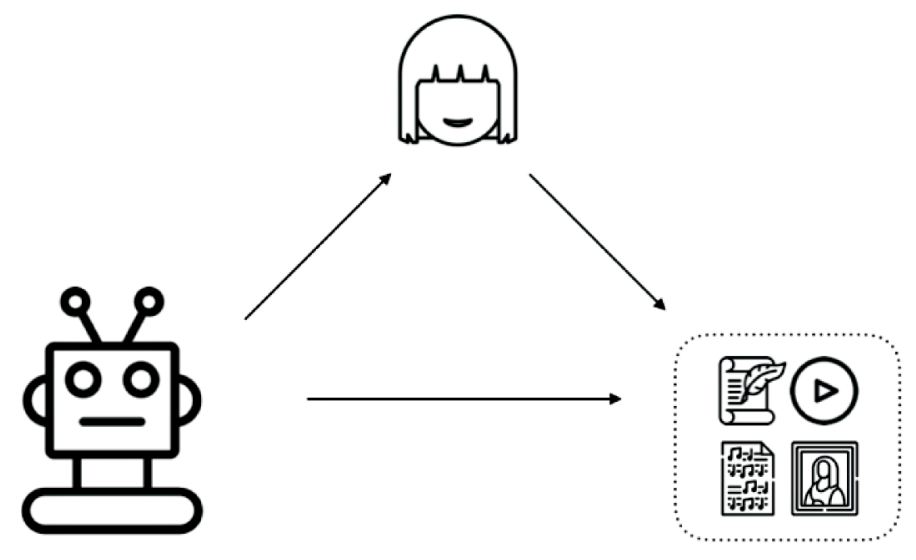

Figure 3. Human as a support for robot creativity

\section{HUMANS AND ROBOTS CO-CREATING: HUMBOTS}

This term «humbot» is the concatenation of «human» and «robot». It is used here to refer to the creative collaboration between a social robot and humans. In this configuration, the robot is a full partner. There is co-creation (see Figure 4).

Robots enter into a feedback loop with humans. An example is a musical band in which one or more players are robot musicians and the others are human (Pan \& Suzuki, 2010; Bretan \& Weinberg, 2016). Each musician may have a solo moment and there is space for improvisation. It is also possible to envision dance choreography with humans and robots, including some improvisational sequences (Peng et al., 2015). In terms of visual arts, certain styles of painting lend themselves to co-creation with human and non-human agents taking turns.

Consider now research on humbots that has been growing since the last two decades. In terms of musical creation, Shimon is an interactive robotic marimba player which improvises in real time with a human pianist, illustrating physical creative interaction (Hoffman \& Winberg, 2011). For dance, FMS (Grace State Machines), a human-machine robotic art performance to music offers an unique close relationship between the human and machine bodies. Finally, the Drawing Operations Unit (DOUG_1), was chosen to contextualize the creative interaction process for a drawing between a robotic arm and the artist Sougwen Chung (Chung, 2019). 


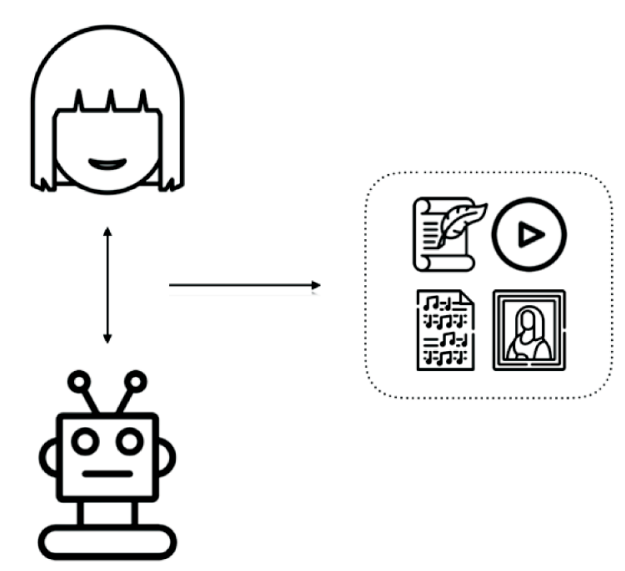

Figure 4. Human-robot (humbot) co-creation

Sandry (2017) developed a framework for thinking about the interactions between humans and machines during the creative process, considering creative interactions as a constant mutual influence happening between humans and machines at different levels. In the beginning, the human interacts with the machine by programming it. Then the human interacts with it in a performative activity. According to this theoretical perspective, these two entities do not have to be seen as equals but need to be analyzed as two creative agents in a cyborg relation, where it is the cyborg ( or humbot) that is creative.

Often studies of humbots remain at a pilot experimental level and only involve nonanthropomorphized robots such as automated arms attached to a drum (Weinberg et al., 2005) or a marimba (Hoffman \& Weinberg, 2011). Pan et al. (2010) set up a musical creation interaction between an anthropomorphized robot and a human. This study shows one of the first successes in the field of creative collaboration thanks to an approach involving the coordination of nonverbal cues (visual and sound). Clues such as the recognition of the direction of the human gaze or the intensity of the sound volume were necessary to have a natural social interaction as we see between two humans

Davis et al. ( 2014) designed another interaction in which robots and humans contribute to the creative process in an embodied model of creativity. In this study, enactive creativity involves a continuous improvisational collaboration between humans and machines. Using perceptual algorithms, an artistic computer robot "colleague" called the Drawing Apprentice could analyze and react to user inputs in real time, in an interactive creative process with a human in the openended creative domain of abstract art. The results of this study confirm the need to explore new theoretical approaches in order to explain more in-depth systems of creative collaboration. Suzuki \& Hashimoto (2004) offer another example in line with the playful interaction in a multi-sensory paradigm of musical and dance performance involving humans and robots

Thus far, humbots have been presented in terms of a coordinated team, with co-action during the productive process. A second mode of collaboration, which can also be considered to be a humbot, involves the division of labor in the creative act (see Figure 5). Robots are quite skilled at 
introducing variability, perhaps through random generation algorithms leading to a large number of works.

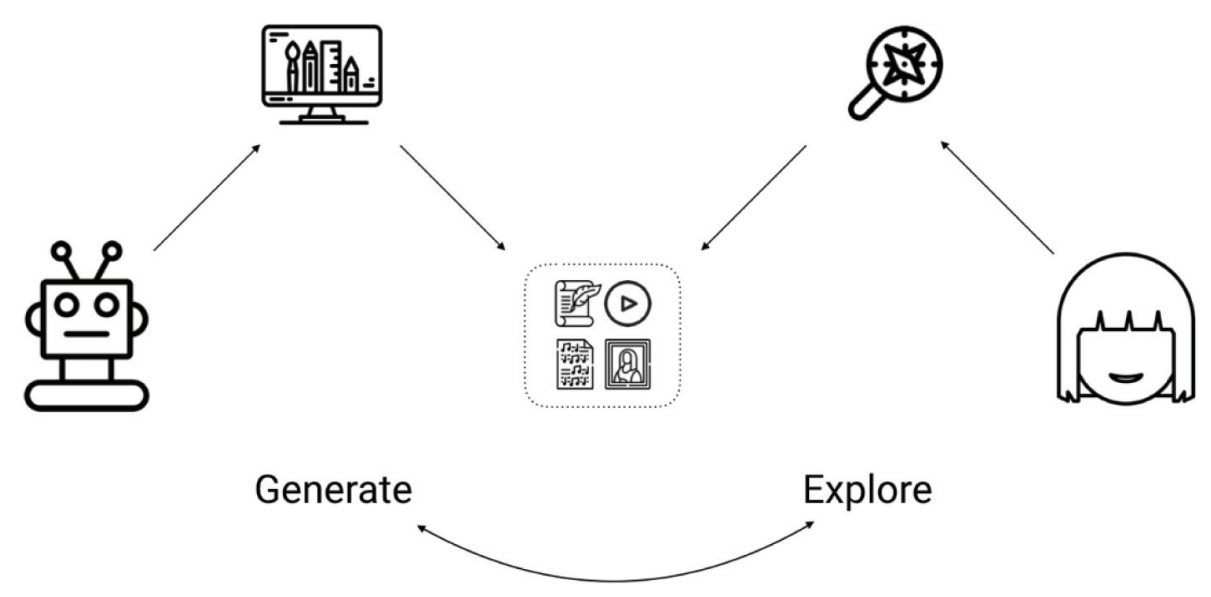

Figure 5. Division of labor in the creative process

The human collaborator enters in the creative process for the evaluation, selection and retention phase. This has, in fact, often been the default case in terms of Al programs that produce artistic work (Hoffman, 2016). The program generates drawings and a human (often the Al programmer in charge of the project) selects the most interesting ones. It is important to recognize this as a humanrobot co-creation.

\section{DISCUSSION AND CONCLUSION}

The three categories proposed in this paper (humans supported by robots, robots supported by humans, and human-robot teams called humbots) are not categorically exclusive. In contrast, one could argue that they mutually complement and enrich each other. For instance, research within a robot-centred HRI perspective is inspired by models of the human creative process and at the same time allows us to inform and refine existing models of creativity.

The opportunities are vast to develop each category. Social robots supporting human creativity is probably the most accessible, and the most socially acceptable avenue for future work. Inspiration can come by examining existing creativity techniques and trying to implement them in social robots. The robots may also estimate the skill level of the human partner, and tailor their input accordingly.

For robot creators, it is likely that more advanced algorithms and machine learning techniques can be applied to robots to boost their creative productive capacity and agency. The main issue is their ability to evaluate and select the promising ideas and implement those. In this case, the robot needs a good representation of the social world in which the productions are supposed to go. This implies a shared understanding of human goals and intentions and the ability to perceive and 
inject meaning into cultural artifacts and social interactions. This kind of development would pave the way to the development of robots with a genuine creative agency.

The third category, collaborative creativity with human and social robot agents, is another rich opportunity. It can expand human creativity as robots may explore unusual problem spaces and are, therefore, more «open-minded». Humans tend to explore a limited conceptual idea space and be affected by so called 'functional fixedness' (Duncker, 1945) due to conventional affordances and rules that they have learned. Artificial agents are likely less constrained, as they have less hidden rules.

The problem of human and artificial creativity transcends the individual human mind and requires multidisciplinary effort and the involvement of all the stakeholders and research communities. The field of human-robot interaction as a highly interdisciplinary area, which intersects robotics, machine learning, engineering, psychology, cognitive science and many others (Dautenhahn, 2007; Fong et al., 2002) represents thus an ideal environment to address the problem. Whereas earlier efforts to tackle the problem of artificial creativity have been concerned with the development of disembodied models based on the "information-processing model of mind" and "Physical Symbol System Hypothesis" (Dautenhahn, 2007; Mitchell, 2021), studies on human-robot interaction extend this research and embed artificial agents into physical context, embracing the distributed and social nature of creativity. As such, social robots could be seen as boundary objects (Arias et al., 2000; Resnick et al. 1991) unifying stakeholders belonging to different communities. We predict that further advances could be achieved soon through the incorporation of computational creativity models in embodied and socially situated artificial agents.

\section{References}

Addesi, A.R., \& Pachet, F. (2005). Experiences with a musical machine: Musical style replication in 3 to 5 year old children. British Journal of Music Education, 22(1), 21-46.

Ali, S., Moroso, T., \& Breazeal, C. (2019). Can children learn creativity from a social robot?. In Proceedings of the 2019 on Creativity and Cognition (pp. 359-368). New York, NY: Association for Computing Machinery.

Alves-Oliveira, P., Arriaga, P., Hoffman, G., \& Paiva, A. (2016). Boosting children's creativity through creative interactions with social robots. In $201611^{\text {th }}$ ACM/IEEE International Conference on Human-Robot Interaction (HRI) (pp. 591-592). IEEE.

Alves-Oliveira, P., Arriaga, P., Paiva, A., \& Hoffman, G. (2019). Guide to build YOLO, a creativity-stimulating robot for children. HardwareX, 6, e00074.

Alves-Oliveira, P., Chandak, A., Cloutier, I., Kompella, P., Moegenburg, P., \& Bastos Pires, AE (2018). Yolo a robot that will make your creativity boom. Companion of the 2018 ACM/IEEE International Conference on Human Robot Interaction (pp. 335-336).

Alves-Oliveira, P., Gomes, S., Chandak, A., Arriaga, P., Hoffman, G., \& Paiva, A. (2020). Software architecture for YOLO, a creativity-stimulating robot. SoftwareX, 11, 100461.

Arias, E., Eden, H., Fischer, G., Gorman, A., \& Scharff, E. (2000). Transcending the Individual Human Mind-Creating Shared Understanding Through Collaborative Design. ACM Transactions on Computer-Human Interaction (TOCHI), 7, 1 (2000), 84-113.

Asada, M., Hosoda, K., Kuniyoshi, Y., Ishiguro, H., Inui, T., Yoshikawa, Y., Ogino, M. \& Yoshida, C. (2009). Cognitive developmental robotics: A survey. IEEE Trans. on Autonomous Mental Development, 1(1), 12-34. 
Belpaeme, T., Kennedy, J., Ramachandran, A., Scassellati, B., \& Tanaka, F. (2018). Social robots for education: A review. Science robotics, 3(21), eaat5954.

Bretan, M., \& Weinberg, G. (2016). A survey of robotic musicianship. Communications of the ACM, 59(5), 100109.

Bruner, J. (1996). The culture of education. Harvard University Press.

Calinon, S., Guenter, F., \& Billard, A. (2005). Goal-directed imitation in a humanoid robot. In Proceedings of the 2005 IEEE International Conference on Robotics and Automation (pp. 299-304). IEEE.

Calinon, S., Li, Z., Alizadeh, T., Tsagarakis, N.G., \& Caldwell, D.G. (2012). Teaching of bimanual skills in a compliant humanoid robot. In IntI Workshop on Human-Friendly Robotics (HFR).

Cangelosi, A. \& Schlesinger, M. (2018). From babies to robots: the contribution of developmental robotics to developmental psychology. Child Development Perspectives, 12(3):183-188.

Chen, H., Park, H.W., \& Breazeal, C. (2020). Teaching and learning with children: Impact of reciprocal peer learning with a social robot on children's learning and emotional engagement. Computers \& Education, $150,103836$.

Chung, S. (2019). Drawing Operations, 2015.

Cropley, A. (2006). In praise of convergent thinking. Creativity Research Journal, 18(3), 391-404.

Csikszentmihalyi, M. (1999). Implications of a systems perspective for the study of creativity. In R. Sternberg (Ed.), Handbook of creativity (pp. 313-335). Cambridge, England: Cambridge University Press.

Dautenhahn, K., \& Billard, A. (1999). Bringing up robots or - the psychology of socially intelligent robots: from theory to implementation. In Proceedings of Autonomous Agents (pp. 366-367). New York, NY: Association for Computing Machinery.

Dautenhahn, K., Ogden, B., \& Quick, T. (2002). From embodied to socially embedded agents - implications for interaction-aware robots. Cognitive Systems Research, 3, 397-428.

Dautenhahn K. (2007). Socially intelligent robots: dimensions of human-robot interaction. Philosophical transactions of the Royal Society of London. Series B, Biological sciences, 362(1480), 679-704.

Davis, N.M., Popova, Y., Sysoev, I., Hsiao, C.P., Zhang, D., \& Magerko, B. (2014). Building Artistic Computer Colleagues with an Enactive Model of Creativity. In ICCC (pp. 38-45).

De Graaf, M.M.A., Allouch, S.B., \& Klamer, T. (2015). Sharing a life with Harvey: Exploring the acceptance of and relationship-building with a social robot. Computers in Human Behavior, 43, 1-14. https://doi. org/10.1016/j.chb.2014.10.030

Duffy, B.R. (2003). Anthropomorphism and the social robot. Robotics and Autonomous Systems, 42(3-4), 177190. https://doi.org/10.1016/s0921-8890(02)00374-3

Engeström, Y. (2001). Expansive learning at work: Toward an activity theoretical reconceptualization. Journal of Education and Work, 14, 133-156.

Engeström, Y., \& Miettinen, R. (1999). Activity theory: A well-kept secret. In Y. Engeström, R. Miettinen, \& R.L. Punamaki (Eds.), Perspectives on activity theory (pp. 1-38). New York: Cambridge University Press.

Fitzgerald, T., Goel, A.K., \& Thomaz, A. (2017). Human-Robot Co-Creativity: Task Transfer on a Spectrum of Similarity. In ICCC (pp. 104-111).

Fong, T., Nourbakhsh, I., \& Dautenhahn K. (2002). A survey of socially interactive robots: concepts,design, and applications. Technical Report No. CMU-RI-TR-02-29. Robotics Institute, Carnegie Mellon University.

Gazeau, J.P., \& Zeghloul, S. (2012). The artist robot: A robot drawing like a human artist. In 2012 IEEE International Conference on Industrial Technology (pp. 486-491). IEEE.

Gibson, J.J. (1986). The ecological approach to visual perception. Hillsdale, NJ: Erlbaum

Glăvenau, V.P. (2012). What can be done with an egg? Creativity, material objects and the theory of affordances. Journal of Creative Behavior, 46, 192-208. https://doi.org/10.1002/jocb.13 
Glăvenau, V.P. (2013). Rewriting the language of creativity: the five A's framework. Review of General Psychology, 17, 69-81. https://doi.org/10.1037/a0029528

Glăveanu, V.P., Hanchett Hanson, M., Baer, J., Barbot, B., Clapp, E.P., Corazza, G.E., ... \& Sternberg, R.J. (2020). Advancing creativity theory and research: A socio-cultural manifesto. The Journal of Creative Behavior, 54(3), 741-745.

Gordon, G., Breazeal, C., \& Engel, S. (2015). Can children catch curiosity from a social robot?. In Proceedings of the Tenth Annual ACM/IEEE International Conference on Human-Robot Interaction (pp. 91-98).

Gubenko, A., Kirsch, C., Smilek, J.N., Lubart, T., \& Houssemand, C. (2021). Educational Robotics and Robot Creativity: An Interdisciplinary Dialogue. Frontiers in Robotics and Al, 8(178).

Guerin, F., Ferreira, P. (2019). Robot Manipulation in Open Environments: New Perspectives. IEEE Transactions on Cognitive and Developmental System, 12(3), (pp. 669-675).

Hoffmann, O. (2016). On Modeling Human-Computer Co-Creativity. In S. Kunifuji, G.A. Papadopoulos, A.M.J. Skulimowski, \& J. Kacprzyk, (Eds.), Knowledge, Information and Creativity Support Systems (pp. 37-48). Cham, Switzerland: Springer International Publishing.

Hoffman, G., \& Weinberg, G. (2011). Interactive improvisation with a robotic marimba player. Autonomous Robots, 31(2-3), 133-153.

Höflich, J.R., \& El Bayed, A. (2015). Perception, Acceptance, and the Social Construction of Robots-Exploratory Studies. Social Robots from a Human Perspective, 39-51. https://doi.org/10.1007/978-3-319-156729_4

Hutchins, E. (1995). Cognition in the wild. Cambridge, MA: MIT Press.

Jones, R.A. (2017). What makes a robot 'social'? Social Studies of Science, 47(4), 556-579.

Kahn Jr, P.H., Kanda, T., Ishiguro, H., Freier, N.G., Severson, R.L., Gill, B.T., ... \& Shen, S. (2012). "Robovie, you'll have to go into the closet now": Children's social and moral relationships with a humanoid robot. Developmental psychology, 48(2), 303.

Kahn, P.H., Kanda, T., Ishiguro, H., Gill, B.T., Shen, S., Ruckert, J.H., \& Gary, H.E. (2016). Human creativity can be facilitated through interacting with a social robot. In 2016 11th ACM / IEEE International Conference on Human Robot Interaction (HRI) (pp. 173-180). IEEE.

Kantosalo, A., Falk, M., \& Jordanous, A. (2021). Embodiment in 18th Century Depictions of Human-Machine Co-Creativity. Frontiers in Robotics and Al, 8(190).

Kato, I., Ohteru, S., Shirai, K., Matsushima, T., Narita, S., Sugano, S., ... \& Fujisawa, E. (1987). The robot musician 'wabot-2' (waseda robot-2). Robotics, 3(2), (pp. 143-155).

Leont'ev, A.N. (1978). Activity, Consciousness, and Personality. Englewood Cliffs, NJ: Prentice Hall.

Leite, I., Martinho, C., \& Paiva, A. (2013). Social Robots for Long-Term Interaction: A Survey. International Journal of Social Robotics, 5(2), 291-308. https://doi.org/10.1007/s12369-013-0178-y

Lindblom, J., \& Ziemke, T. (2003), Social situatedness of natural and artificial intelligence: Vygotsky and beyond. Adaptive Behavior, 11, 79-96.

Luo, RC, \& Liu, YJ (2018). Robot Artist Performs Cartoon Style Facial Portrait Painting. In 2018 IEEE / RSJ International Conference on Intelligent Robots and Systems (IROS) (pp. 7683-7688). IEEE.

Lungarella, M., Metta, G., Pfeifer, R., \& Sandini, G. (2004). Developmental robotics: a survey. Connection science, 15(4), 151-190.

MacDorman, K.F. (2019). La Vallée de l'Étrange de Mori Masahiro. e-Phaïstos, (VII-2). https://doi.org/10.4000/ ephaistos.5333

Magnenat-Thalmann, N., Yuan, J., Thalmann, D., \& You, B.-J. (Eds.). (2016). Context Aware Human-Robot and Human-Agent Interaction. Human-Computer Interaction Series. https://doi.org/10.1007/978-3-31919947-4

Min, H., Yi, C., Luo, R., Zhu, J. \& Bi, S. (2016). Affordance research in developmental robotics: A survey. IEEE Transactions on Cognitive and Developmental Systems, 8(4), 237-255. 
Mitchell, M. (2021). Why Al is Harder Than We Think. In: arXiv: 2104.12871

Mori, M., MacDorman, K., \& Kageki, N. (2012). The Uncanny Valley [From the Field]. IEEE Robotics \& Automation Magazine, 19(2), 98-100. https://doi.org/10.1109/mra.2012.2192811

OECD. (2019). OECD future of education and skills 2030-Conceptual learning framework-Concept note: Student agency for 2030. Paris: Editions OECD.

OECD (2021). OECD Digital Education Outlook 2021 : Pushing the Frontiers with Artificial Intelligence, Blockchain and Robots. Paris: Editions OECD. https://doi.org/10.1787/589b283f-en

Oliveira, P.A. (2020). Boosting children's creativity through creative interactions with social robots. https://www. researchgate.net/publication/348293686_Boosting_Children's_Creativity_through_Creative_Interactions_with_Social_Robots

Otero, N., Saunders, J., Dautenhahn, K., \& Nehaniv, C.L. (2008). Teaching robot companions: the role of scaffolding and event structuring. Connection Science, 20(2-3), 111-134.

Pachet, F. (2003). The continuator: Musical interaction with style. Journal of New Music Research, 32(3), 333341.

Pakrasi, I., Chakraborty, N., Cuan, C., Berl, E., Rizvi, W., \& LaViers, A. (2018). Dancing droids: an expressive layer for mobile robots developed within choreographic practice. In International Conference on Social Robotics (pp. 410-420). Springer, Cham.

Pan, Y., Kim, MG, \& Suzuki, K. (2010). A Robot Musician Interacting with a Human Partner through Initiative Exchange. In NIME (pp. 166-169).

Park, H.W., Rosenberg-Kima, R., Rosenberg, M., Gordon, G., \& Breazeal, C. (2017). Growing growth mindset with a social robot peer. In Proceedings of the 2017 ACM/IEEE international conference on human-robot interaction (pp. 137-145).

Park, H.W., Grover, I., Spaulding, S., Gomez, L., \& Breazeal, C. (2019). A model-free affective reinforcement learning approach to personalization of an autonomous social robot companion for early literacy education. In Proceedings of the AAAI Conference on Artificial Intelligence (Vol. 33, No. 01, pp. 687-694).

Peng, H., Zhou, C., Hu, H., Chao, F., \& Li, J. (2015). Robotic dance in social robotics - a taxonomy. IEEE Transactions on Human-Machine Systems, 45(3), 281-293.

Resnick, L.B., Levine, J.M., \& Teasley, S.D. (Eds.). (1991). Perspectives on socially shared cognition. American Psychological Association.

Runco, M.A., \& Jaeger, G.J. (2012). The standard definition of creativity. Creativity Research Journal, 24(1), 92-96.

Russell, S.J., \& Norvig, P. (2010). Artificial Intelligence-A Modern Approach (3. internat. ed.). Pearson Education.

Saerbeck, M., Schut, T., Bartneck, C., \& Janse, M.D. (2010, April). Expressive robots in education: varying the degree of social supportive behavior of a robotic tutor. In Proceedings of the SIGCHI conference on human factors in computing systems, CHI 10 (pp. 1613-1622).

Sandry, E. (2017). Creative collaborations with machines. Philosophy \& Technology, 30(3), 305-319.

Smith, G. F. (1998). Idea-generation techniques: A formulary of active ingredients. The Journal of Creative Behavior, 32(2), 107-134.

Smith L.B., \& Gasser M. (2005). The development of embodied cognition: six lessons from babies. Artificial Life, 11, 13-30.

Suzuki, K., \& Hashimoto, S. (2004). Robotic interface for embodied interaction via dance and musical performance. Proceedings of the IEEE, 92(4), 656-671.

Thrun, S., Hähnel, D., Ferguson, D., Montemerlo, M., Triebel, R., Burgard, W., Baker, C., Omohundro, Z., Thayer, S., \& Whittaker, W. (2003). A system for volumetric robotic mapping of abandoned mines. In 2003 IEEE International Conference on Robotics and Automation. 3/(10) (pp. 4270-4275).

Thrun, S. (2004). Toward a framework for human-robot interaction. Human-Computer Interaction, 19(1-2), 9-24. 
Toubia, O., Berger, J., \& Eliashberg, J. (2021). How quantifying the shape of stories predicts their success. Proceedings of the National Academy of Sciences, 118(26).

Tresset, P., \& Leymarie, FF (2013). Portrait drawing by Paul the robot. Computers \& Graphics, 37(5), 348-363.

U.N. and I.F.R.R. (2002). United Nations and The International Federation of Robotics. World Robotics 2002. New York-Geneva: United Nations.

Ventura, D. (2016, June). Mere generation: Essential barometer or dated concept. In Proceedings of the Seventh International Conference on Computational Creativity (pp. 17-24). Paris: Sony CSL.

Weinberg, G., Driscoll, S., \& Parry, M. (2005, August). Musical interactions with a perceptual robotic percussionist. In ROMAN 2005. IEEE International Workshop on Robot and Human Interactive Communication, 2005 (pp. 456-461). IEEE.

Zagoruyko, S., \& Komodakis, N. (2015). Learning to compare image patches via convolutional neural networks. In Proceedings of the IEEE conference on computer vision and pattern recognition (pp. 4353-4361).

Zlatev, J. (2001). The epigenesis of meaning in human beings, and possibly in robots. Minds and Machines, $11,155-195$.

(c) Copyright by Faculty of Education, University of Bialystok,

20 Swierkowa St., 15-328 Bialystok, Poland

tel. +48857457283

e-mail: creativity@uwb.edu.pl

http://www.creativity.uwb.edu.pl 